\title{
Another Look at the Method of Y-Standardization in Logit and Probit Models
}

Karlson, Kristian Bernt

Published in:

Journal of Mathematical Sociology

DOI:

$10.1080 / 0022250 X .2014 .897950$

Publication date:

2015

Citation for published version (APA):

Karlson, K. B. (2015). Another Look at the Method of Y-Standardization in Logit and Probit Models. Journal of Mathematical Sociology, 39(1), 29-38. https://doi.org/10.1080/0022250X.2014.897950 


\title{
Another Look at the Method of Y-Standardization in Logit and Probit Models
}

\author{
Kristian Bernt Karlson (kbk@soc.ku.dk)
}

THIS PAPER IS PUBLISHED IN

JOURNAL OF MATHEMATICAL SOCIOLOGY 2015

VOL. 39, NO.1, 29-38

Link: http://www.tandfonline.com/doi/full/10.1080/0022250X.2014.897950

This is a post-print (i.e. final draft post-refereeing) version according to SHERPA/ROMEO

Revised version: 12-DEC-2013

Department of Sociology, University of Copenhagen, Øster Farigmagsgade 5, Building 16, DK-1014 Copenhagen K, Denmark

Words: 3,585

Running head: The Method of Y-Standardization

Key words: logit, logistic, probit, comparison, identification, rescaling, attenuation, standardization, y-standardized, y-standardization 


\title{
Another Look at the Method of Y-Standardization in Logit and Probit Models
}

\begin{abstract}
This paper takes another look at the derivation of the method of Y-standardization used in sociological analyses involving comparisons of coefficients across nested logit or probit models. It shows that the method can be derived under less restrictive assumptions than hitherto suggested. Rather than assuming that the logit or probit fixes the variance of the latent error at a known constant, it suffices to assume that the variance of the error is unknown. A further result suggests that using Y-standardization for cross-model comparisons is likely to be biased by model differences in the fit of the latent error to the assumed logistic or normal distribution. Under correct specification Y-standardization recovers an effect size metric similar to Cohen's d.
\end{abstract}




\section{Another Look at the Method of Y-Standardization in Logit and Probit Models}

\section{INTRODUCTION}

Sociologists routinely compare coefficients of the same variable across regression models successively including control variables or blocks of control variables (Clogg et al. 1995). Changes in the coefficients inform the researcher about confounding or mediating relationships central to much sociological inquiry. However, in nonlinear probability models such as the logit or probit, such comparisons are distorted by a rescaling or attenuation bias. Adding control variables to a logit or probit model will increase the magnitude of the logit or probit coefficient, even if the control variables are uncorrelated with the original predictor variables (Gail et al. 1984; Greenland 1987; Greenland et al. 1999; Hauck et al. 1991; Yatchew and Griliches 1985; Winship and Mare 1984; Wooldridge 2002).

The attenuation bias stems from the scale identifiability of logit or probit coefficients. These coefficients are identified up to a scale, which is a function of the conditional variance of the latent outcome variable assumed to underlie the logit or probit model. Adding control variables to the logit or probit model will reduce the error variance, which in turn leads to increases in the magnitude of the estimated logit or probit coefficient over and above any change in coefficients caused by true confounding. Consequently, logit or probit coefficients of the same predictor variable in models with different covariates are not directly comparable.

To make coefficients comparable across logit or probit models, social researchers routinely use the method of Y-standardization to rescale coefficients of the different models to the standard deviation of the latent outcome variable, giving the coefficients an interpretation equivalent to coefficients standardized on the outcome variable in linear models. The approach originates in McKelvey and Zavoina's (1975) seminal work on the 
ordered probit model. Winship and Mare $(1983,1984)$ suggested using the method for comparing coefficients of logit or probit models across same-sample nested models. Long (1997) coined these coefficients Y-standardized, with Y referring to the latent outcome variable. Today the method of Y-standardization is widely used in sociological research involving comparisons of logit or probit coefficients across same-sample nested models.

However, in previous research the method of Y-standardization appears to have been derived from a somewhat restrictive assumption placed on the error term of the latent outcome variable assumed to underlie the logit or probit model. The variance of the error term is assumed to equal $\pi^{2} / 3$ for the logit and 1 for probit (Winship and Mare 1984; Long 1997). In this paper, I argue that a less restrictive assumption can be maintained without loss of generality. Rather than assuming that the variance of the latent error is fixed at a known constant, it suffices to assume that the latent error follows a logistic (for the logit model) or normal (for the probit model) distribution with unknown variance-an assumption that also appears to have been maintained in the seminal work by McKelvey and Zavoina (1975).

Under the less restrictive assumption of unknown variance, it is possible to obtain the Y-standardized coefficient and to retain the interpretation of the Y-standardized coefficient as a change in standard deviations in the latent outcome variable for a unit change in the predictor variable. Thus there is little need for placing the additional assumption of known error variance in the derivation of the method of Y-standardization. Maintaining the less restrictive assumption of unknown variance of the latent error also has another advantage in terms of model interpretation: Consistent with the interpretation in linear models, adding covariates to a logit or probit model will reduce the variance of the latent error term, something that would not be possible if the error variance always was fixed at the same, known constant. 
This paper derives the method of Y-standardization under the less restrictive assumption of unknown variance of the latent error term. It further argues that comparing Ystandardized coefficients across models should be considered an approximation, because such comparisons are affected by differences in the fit of the conditional variances of the latent outcomes in the different models to the assumed logistic or normal distribution. The paper closes by briefly noticing that under correct specification the Y-standardized coefficient can be interpreted as an effect size metric akin to Cohen's d.

\section{A LATENT VARIABLE MODEL}

In this section, I derive the logit and probit model from a linear model involving a latent outcome variable. To keep the derivations simple, I assume that the model only includes a single predictor variable. The Appendix gives derivations for the case with multiple predictor variables.

Let $\mathrm{Y}^{*}$ be a latent, continuous outcome variable, and $\mathrm{X}$ an observed predictor variable. Assume that they are linearly related:

$$
Y^{*}=\alpha+\beta X+\varepsilon
$$

where $\varepsilon$ is a random error term. To derive the logit model, I impose a distributional assumption on $\varepsilon$ : It follows a logistic distribution with mean zero and variance $\sigma_{\varepsilon}^{2}$. Similarly, to derive the probit model, I assume that $\varepsilon$ follows a normal distribution with mean zero and variance $\sigma_{\varepsilon}^{2}$. Given either of these assumptions, I rewrite $\varepsilon$ as

$$
\varepsilon=s \omega
$$

where $\omega$, for the logit, is a standard logistic random variable with mean zero and variance $\pi^{2} / 3$ and, for the probit, is a standard normal random variable with mean zero and variance 
1. $s$ is a scale parameter which allows the variance in $\varepsilon$ to differ from that of the standard logistic or normal distribution. Thus the variance in $\varepsilon$ can be written as

$$
V(\varepsilon)=V\left(Y^{*} \mid X\right)=s^{2} V(\omega),
$$

where $V(\omega)=\frac{\pi^{2}}{3}$ for the logit and $V(\omega)=1$ for the probit. In other words, this derivation assumes that the distribution of the latent error term in (1) is nothing but a multiple of a standard distribution with known mean and variance. But the derivation does not assume that the error variance is fixed at a known number; the scale parameter, $s$, allows the error variance to differ from the variance of the standard random variable, $\omega$. This result is also evident from Equation 2.5 in McKelvey and Zavoina's (1975:105-106) derivation of the ordered probit model. ${ }^{1}$ The derivation nevertheless differs from that reported in Winship and Mare (1984:517) and Long $(1997: 42,47,71,122)$ in which the error variance in (3) is assumed to equal a constant ( $\pi^{2} / 3$ for the logit, 1 for the probit). As we will see, there is no need for maintaining such restrictive assumption. The Y-standardized coefficient can be obtained under the less restrictive assumption of unknown variance of the latent error, as stated in (3).

To complete the derivation of the logit and probit models, assume that we observe a binary outcome variable, $\mathrm{Y}$, which is related to $\mathrm{Y}^{*}$ by the following threshold rule:

$$
\begin{aligned}
& Y=1 \text { if } Y^{*}>\tau \\
& Y=0 \text { otherwise. }
\end{aligned}
$$

For the logit it then follows that

$$
\operatorname{Pr}\left(Y^{*}>\tau\right)=\operatorname{Pr}\left(\varepsilon>-\left[\frac{\alpha-\tau+\beta X}{s}\right]\right)=\frac{\exp \left(\frac{\alpha-\tau}{s}+\frac{\beta}{s} X\right)}{1+\exp \left(\frac{\alpha-\tau}{s}+\frac{\beta}{s} X\right)}
$$

which is the familiar logistic response model,

\footnotetext{
${ }^{1}$ For similar derivations, see Amemiya (1981), Maddala (1983), and Powers and Xie (2000:56-58).
} 


$$
\operatorname{logit}\left[\operatorname{Pr}\left(Y^{*}>\tau\right)\right]=a+b X
$$

Similarly, we can derive the probit model,

$$
\operatorname{Pr}\left(Y^{*}>\tau\right)=\Phi\left[\frac{\alpha-\tau}{s}+\frac{\beta}{s} X\right]=\Phi(a+b X),
$$

where $\Phi(\cdot)$ is the normal cdf.

In (5b) and (6), $b=\frac{\beta}{s}$, meaning that the coefficient of $X$ in a logit or probit model identifies the coefficient, $\beta$, in the linear model in (1) up to a unknown scale parameter, $s .^{2}$ This result is identical to that reported in Equation (2.8) in McKelvey and Zavoina (1975:106).

\section{THE METHOD OF Y-STANDARDIZATION}

The method of Y-standardization can be derived under the less restrictive assumption that the variance of the latent error is unknown. To derive the method of Y-standardization, I decompose the variance in $\mathrm{Y}^{*}$,

$$
V\left(Y^{*}\right)=\beta^{2} V(X)+V\left(Y^{*} \mid X\right)=\beta^{2} V(X)+s^{2} V(\omega)
$$

where $V(\omega)=\pi^{2} / 3$ for the logit and $V(\omega)=1$ for the probit, and where the second equality holds given (3). Because $\beta=b s$, I can rewrite (7a) as

$$
V\left(Y^{*}\right)=b^{2} s^{2} V(X)+s^{2} V(\omega)=s^{2}\left[b^{2} V(X)+V(\omega)\right]
$$

which means that the variance in $Y^{*}$ equals the sum of the variance of the linear predictor of the logit or probit and the variance of the standard logistic or normal random variable $\left(\pi^{2} / 3\right.$ or 1) multiplied by the squared scale parameter.

\footnotetext{
${ }^{2}$ The intercept is a composite of the intercept in (1), the threshold parameter in (4), and the scale parameter. The intercept is irrelevant for the derivations reported here and I ignore it throughout.
} 
The expression for the variance in (7b) differs from that reported in Long $(1997: 70,129,254)$, who, using the notation of this paper, writes the variance in $\mathrm{Y}^{*}$ as

$$
V\left(Y^{*}\right)=b^{2} V(X)+V(\omega) .
$$

This expression of the variance holds under the assumption that the error variance is fixed at $\pi^{2} / 3$ for the logit or 1 for the probit, and it differs from that in ( $7 b$ ) by the squared scale parameter. However, to derive the Y-standardized coefficient, there is no need to maintain the restrictive assumption of a fixed, known error variance. As we will see, it suffices to assume unknown error variance.

To derive the Y-standardized coefficient from the less restrictive assumption of unknown error variance, as stated in (7b), I first define the Y-standardized logit or probit coefficient as

$$
b^{Y S T D}=\frac{\beta}{S D\left(Y^{*}\right)}=\frac{\operatorname{COV}\left(X, Y^{*}\right)}{V(X) S D\left(Y^{*}\right)} .
$$

Notice how this coefficient is identical to coefficients standardized on the outcome in linear models. The only difference is that $Y^{*}$ is latent. However, I can easily obtain (9a) from estimatable quantities,

$$
b^{Y S T D}=\frac{\beta}{S D\left(Y^{*}\right)}=\frac{b s}{\sqrt{s^{2}\left[b^{2} V(X)+V(\omega)\right]}}=\frac{b}{\sqrt{b^{2} V(X)+V(\omega)}},
$$

using the rule that $\beta=b s$ and, using (7b), that $S D\left(Y^{*}\right)=s \sqrt{b^{2} V(X)+V(\omega)}$. Notice that the scale parameter, $s$, cancels to obtain an expression including only estimatable quantities. The expression in (9b) also suggests that, under the assumption of unknown error variance, the Ystandardized coefficient is not obtained by dividing by the standard deviation of the latent variable-something that only would be true under the assumption of known variance as stated in (8) (Long 1997; Long and Freese 2006). 
In sum, (9b) shows the Y-standardized coefficient derived from an assumption of unknown variance of the latent error, an assumption less restrictive than assuming known variance. Assuming unknown error variance in the derivation of the logit or probit has another advantage in terms of model interpretation. Similar to linear regression models, adding covariates to the model will reduce the variance of the latent error, something that could not be true if the variance always was fixed at the same, known constant. However, in contrast to linear models, adding these covariates will reduce the magnitude of the scale parameter, leading to the rescaling or attenuation bias described in the methodological literature.

\section{EXAMPLE}

To illustrate the subtle difference between the derivations assuming known and unknown error variance, I simulated two data sets. Both data sets include two predictor variables, $\mathrm{X}$ and $\mathrm{Z}$, and a random error term, all normally distributed with mean zero and unit variance. In both data sets $\mathrm{X}$ and $\mathrm{Z}$ are orthogonal, meaning that adding $\mathrm{Z}$ to the equation cannot confound the effect of $\mathrm{X}$ in the conventional meaning of the term. In the first dataset, the true model is

$$
Y^{*}=X+2 Z+\varepsilon
$$

whereas in the second data set, the true model is

$$
Y^{*}=X+Z+2 \varepsilon
$$

yielding a common variance in $\mathrm{Y}^{*}$ in both data sets, namely $V\left(Y^{*}\right)=6$. The two datagenerating models differ by the fraction of the total variance in $\mathrm{Y}^{*}$ that is a result of the error variance. This setup should affect the estimated coefficients, but not the Y-standardized counterparts, because of the common variance in $\mathrm{Y}^{*}$ and because the true coefficients of $\mathrm{X}$ 
are the same in the two models. In the simulated data, to increase precision, I randomly draw $\mathrm{N}=100,000$ observations.

Assuming that $Y$ is a binary manifestation of $Y^{*}$, such that $\mathrm{Y}=1$ if $Y^{*}>0$ and $\mathrm{Y}=0$ otherwise, in Table 1 I report unstandardized and Y-standardized coefficients using probit models. In both panels I compare the coefficients of $\mathrm{X}$ without and with control for $\mathrm{Z}$ and report both unstandardized and Y-standardized coefficients. Comparing the unstandardized coefficients of $\mathrm{X}$ in $\mathrm{M} 1$ and $\mathrm{M} 2$ in both panels suggests that $\mathrm{Z}$ is a suppressor, increasing the magnitude of the effect of $\mathrm{X}$. This apparent suppressor effect is much stronger in panel A (in which adding $\mathrm{Z}$ has a much larger impact on reducing the error variance). However, the $\mathrm{Y}$ standardized coefficients are, except for rounding, identical across both panels and all four models, suggesting that the apparent suppressor effects are an artifact of the attenuation bias. This result is what we would have expected given the model specifications in Models (A) and (B).

\section{--TABLE 1 HERE --}

Yet the two panels in Table 1 differ in one important regard. Using the variance formula in (8) - i.e., that derived from the assumption that the latent error variance is fixed at one-yields quite different estimates in Models M2 and M4. In M2, the variance estimate is 5.894 which is very close to the true variance of 6 , whereas in M3, the corresponding estimate of 1.489 is notably smaller. But this difference cannot be a result of differing variances in $\mathrm{Y}^{*}$, which is fixed by design. Thus the difference is an artifact of the assumption, being incorrect in Panel B, that the latent error variance equals one. ${ }^{3}$ Nevertheless, despite these differences, the Y-standardized coefficients of X are still correctly recovered in all models, thereby illustrating that there is no need to assume a fixed, known

\footnotetext{
${ }^{3}$ In fact, according to the equation in (7b), the variances of $\mathrm{Y}^{*}$ in Panels A and B roughly differ by a factor of 4, being the ratio of the respective squared scale parameters in Models (A) and (B).
} 
variance to obtain the Y-standardized coefficients. Maintaining the less restrictive assumption of unknown error variance suffices.

\section{CROSS-MODEL COMPARISONS}

Correct specification of logit or probit models relies on the extent to which the error term in (1) follows the assumed logistic or normal distribution (Horowitz and Savin 2001; Cramer 2007). This assumption has consequences for the use of Y-standardization in cross-model comparisons, the very purpose for which Y-standardization was suggested as a solution (Winship and Mare 1984). Whenever a researcher estimates two or more logit or probit models and Y-standardizes the coefficients for comparison purposes, the models differ not only in their residual scaling but also in the fit of the latent error to the assumed logistic or normal distribution. This difference in latent error fit depends on the distribution of the covariates that are sequentially added to the model.

For example, in a model including only $\mathrm{X}$ the error is a mixture of omitted $\mathrm{Z}$ and the error term in the model including Z. Even if the error in the model including $\mathrm{Z}$ is logistic or normal — thereby being correctly specified — the error in the model excluding $\mathrm{Z}$ will rarely be logistic or normal. ${ }^{4}$ It will depend on the particular distribution of Z (Neuhaus et al. 1991). Consequently, the method of Y-standardization should be considered an approximation. While it corrects for rescaling bias in same-sample cross-model comparisons, it is affected by model differences in the fit of the latent error to the assumed logistic or normal distribution.

Using Monte Carlo simulations, Karlson et al. (2012) found that the method of Ystandardization exhibits bias in cross-model coefficient comparisons whenever the distribution of $\mathrm{Z}$ is very different from that of the error term in a model including both $\mathrm{X}$ and

\footnotetext{
${ }^{4}$ In the probit this condition might be met whenever $\mathrm{Z}$ and the error in the model including $\mathrm{Z}$ are normal (Yatchew and Griliches 1985). However, the condition is unlikely to be met in applied work.
} 
Z. Karlson et al. (2012) develop a method that holds constant both scaling and fit of the latent error to the assumed distribution, thereby overcoming the issue pertaining to the method of Y-standardization.

\section{EFFECT SIZE METRIC}

The expression in (9) gives an interpretation to the Y-standardized coefficient that, as argued by Breen and Karlson (2013), might be useful in certain areas of social research. Whenever X is a binary variable - a treatment indicator-the Y-standardized coefficient equals the effect size metric, Cohen's d (Cohen 1969), on the latent outcome variable,

$$
b^{Y S T D}=\frac{\operatorname{COV}\left(X, Y^{*}\right)}{V(X) S D\left(Y^{*}\right)}=\frac{E\left(Y^{*} \mid X=1\right)-E\left(Y^{*} \mid X=0\right)}{S D\left(Y^{*}\right)},
$$

which easily extends to the case in which the treatment effect is conditional on other covariates, Z,

$$
b_{X \mid Z}^{Y S T D}=\frac{\operatorname{COV}\left(X, Y^{*} \mid Z\right)}{V(X \mid Z) S D\left(Y^{*}\right)}=\frac{E\left(Y^{*} \mid X=1, Z\right)-E\left(Y^{*} \mid X=0, Z\right)}{S D\left(Y^{*}\right)}
$$

Both effect size metrics are directly obtainable from the formula in (9b) (see also the Appendix). The results in (10) depends on the identifying distributional assumption imposed on the latent error term. Whenever researchers have reasons for believing that this assumption does not hold, the coefficients in (10) should be considered approximations.

\section{CONCLUSION}

The method of Y-standardization standardizes coefficients in logit or probit models on the standard deviation of the latent outcome, $\mathrm{Y}^{*}$, assumed to underlie the binary, observed outcome variable, Y. Y-standardized coefficients are unaffected by the rescaling or attenuation bias inherent the cross-model comparisons of logit and probit coefficients. They 
are therefore highly useful in sociological research in which nonlinear probability models are very popular. In contrast to previous research on this topic, this paper shows that the Ystandardized coefficients can be derived without assuming that the variance of the latent error of the model underlying the logit or probit model is fixed at a known constant. It is sufficient to assume that the variance is unknown, a result that appears to be consistent with that reported in McKelvey and Zavoina (1975). Consequently, there is little need for placing the additional assumption of known error variance in the derivation of the method of $\mathrm{Y}$ standardization.

Further results suggest that in coefficient comparisons across sample-sample nested models, Y-standardization is likely to be affected by model differences in the fit of the latent error to the assumed logistic or normal distribution—something that can introduce bias into the comparisons. Finally, under correct specification, Y-standardization recovers an effect size similar to Cohen's d. The results presented in this paper easily extend to the ordered and multinomial case and to other nonlinear probability models and possibly to the class of generalized linear models.

\section{APPENDIX: DERIVATION USING MULTIPLE PREDICTORS}

This appendix derives the method of Y-standardization for the multiple regression case. Assume that there are $k=1,2,3, \ldots, K$ predictor variables. I then write the latent linear model as

$$
Y^{*}=\alpha+\mathbf{X}^{\prime} \boldsymbol{\beta}+s \omega=\alpha+\beta_{1} X_{1}+\beta_{2} X_{2}+\ldots+\beta_{K} X_{K}+s \omega
$$

and maintain the same assumptions on $\omega$ as in the main text. Under the threshold assumption in (4), the logit and probit models are

$$
\operatorname{logit}\left[\operatorname{Pr}\left(Y^{*}>\tau\right)\right]=a+\mathbf{X}^{\prime} \mathbf{b}
$$




$$
\operatorname{Pr}\left(Y^{*}>\tau\right)=\Phi\left(a+\mathbf{X}^{\prime} \mathbf{b}\right)
$$

where $\mathbf{b}=s^{-1} \boldsymbol{\beta}$. The variance in $\mathrm{Y}^{*}$ can be written as

$$
V\left(Y^{*}\right)=\boldsymbol{\beta}^{\prime} \boldsymbol{\Sigma}_{\mathbf{x}} \boldsymbol{\beta}+V\left(Y^{*} \mid X\right)=s^{2}\left[\mathbf{b}^{\prime} \boldsymbol{\Sigma}_{\mathbf{x}} \mathbf{b}+V(\omega)\right]
$$

where $\boldsymbol{\Sigma}_{\mathbf{X}}$ is the variance-covariance matrix of the $K$ predictor variables, and $\boldsymbol{\beta}^{\prime} \boldsymbol{\Sigma}_{\mathbf{X}} \boldsymbol{\beta}$ is the variance of the linear prediction of the underlying model and $\mathbf{b}^{\prime} \boldsymbol{\Sigma}_{\mathbf{X}} \mathbf{b}$ is the corresponding variance of the logit or probit model. The Y-standardized coefficient for the $k^{\prime}$ th variable is then given by

$$
b_{k}^{Y S T D}=\frac{\beta_{k}}{S D\left(Y^{*}\right)}=\frac{b_{k} s}{\sqrt{s^{2}\left[\mathbf{b}^{\prime} \Sigma_{\mathbf{x}} \mathbf{b}+V(\omega)\right]}}=\frac{b_{k}}{\sqrt{\mathbf{b}^{\prime} \Sigma_{\mathbf{x}} \mathbf{b}+V(\omega)}},
$$

where the latter expression includes only estimatable quantities. Since $\sqrt{\mathbf{b}^{\prime} \boldsymbol{\Sigma}_{\mathbf{X}} \mathbf{b}+V(\omega)}$ is a scalar, it follows that the vector of Y-standardized coefficient can be obtained from

$$
\mathbf{b}^{\text {YSTD }}=r \cdot \mathbf{b},
$$

where $r=\left[\mathbf{b}^{\prime} \boldsymbol{\Sigma}_{\mathbf{x}} \mathbf{b}+V(\omega)\right]^{-1 / 2}$.

\section{REFERENCES}

Amemiya, T. (1981). Qualitative Response Models: A Survey. Journal of Econometric Literature, 19, 1483-1536.

Breen, R. and Karlson, K.B. (2013). Counterfactual Causal Analysis and Non-Linear Probability Models. Pp. 167-188 in Handbook of Causal Analysis for Social Research (ed S.L. Morgan). New York: Springer. 
Cohen, J. (1969). Statistical Power Analysis for the Behavioral Sciences. New York: Academic Press.

Clogg, C.C., Petkova, E., and Haritou, A. (1995). Statistical Models for Comparing Regression Coefficients between Models. The American Journal of Sociology, 100, 12611293.

Cramer, J.S. (2003). Logit Models. From Economics and Other Fields. Cambridge: Cambridge University Press.

Cramer, J.S. (2007). Robustness of Logit Analysis: Unobserved Heterogeneity and Misspecified Disturbances. Oxford Bulletin of Economics and Statistics, 69, 545-555.

Gail, M.H., Wiand, S., and Piantadosi, S. (1984). Biased Estimates of Treatment Effect in Randomized Experiments with Nonlinear Regressions and Omitted Covariates. Biometrika, $71,431-444$.

Greenland, S. (1987). Interpretation and Choice of Effect Measures in Epidemiological Analysis. American Journal of Epidemiology, 125, 761-768.

Greenland, S., Robins, J.M., and Pearl, J. (1999). Confounding and Collapsibility in Causal Inference. Statistical Science, 14, 29-46. 
Hauck,W.W., Neuhaus, J.M., Kalbfleish, J.D., and Anderson, S. (1991). A Consequence of Omitted Covariates When Estimating Odds Ratios. Journal of Clinical Epidemiology, 44, 7781.

Horowitz, J.L. and Savin, N.E. (2001). Binary Response Models: Logits, Probits and Semiparametrics. The Journal of Economic Perspectives, 15, 43-56.

Long, J.S. (1997). Regression Models for Categorical and Limited Dependent Variables. Thousand Oaks: Sage.

Karlson, K.B., Holm, A., and Breen, R. (2012). Comparing Regression Coefficients Between Models using Logit and Probit: A New Method. Sociological Methodology, 42, 286-313.

Maddala, G.S. (1983). Limited-dependent and qualitative variables in econometrics. New York: Cambridge University Press.

McKelvey, R.D. and Zavoina, W. (1975). A Statistical Model for the Analysis of Ordinal Level Dependent Variables. Journal of Mathematical Sociology, 4, 103-120.

Neuhaus, J.M., Kalbfleisch, J.D., and Hauck, W.W. (1991). A Comparison of ClusterSpecific and Population-Averaged Approaches for Analyzing Correlated Binary Data. International Statistical Review, 59, 25-35. 
Powers, D.A. and Xie, Y. (2000). Statistical Methods for Categorical Data Analysis. San Diego, California: Academic Press.

Winship, C. and Mare, R.D. (1983). Structural Equations and Path Analysis for Discrete Data. The American Journal of Sociology, 89, 54-110.

Winship, C. and Mare, R.D. (1984). Regression Models with Ordinal Variables. American Sociological Review, 49, 512-525.

Wooldridge, J.M. (2002). Econometric analysis of cross section and panel data. Cambridge, MA: MIT Press.

Yatchew, A. and Griliches, Z. (1985). Specification Error in Probit Models. The Review of Economics and Statistics, 67, 134-139. 
Table 1. Unstandardized and Y-standardized probit coefficients. Simulated data.

\begin{tabular}{|c|c|c|c|c|}
\hline \multicolumn{5}{|l|}{ PANEL A: $Y^{*}=X+2 Z+\varepsilon$} \\
\hline & \multicolumn{2}{|c|}{ M1 } & \multicolumn{2}{|c|}{ M2 } \\
\hline & $b$ & $b^{Y S T D}$ & $b$ & $b^{Y S T D}$ \\
\hline $\mathrm{X}$ & 0.443 & 0.405 & 0.985 & 0.406 \\
\hline $\mathrm{Z}$ & & & 1.987 & 0.818 \\
\hline $\begin{array}{l}\text { Estimated variance of } \mathrm{Y}^{*} \\
\text { according to }(7 \mathrm{~b})\end{array}$ & \multicolumn{2}{|c|}{1.194} & \multicolumn{2}{|c|}{5.894} \\
\hline \multicolumn{5}{|l|}{ PANEL B: $Y^{*}=X+Z+2 \varepsilon$} \\
\hline & \multicolumn{2}{|c|}{ M3 } & \multicolumn{2}{|c|}{ M4 } \\
\hline & $b$ & $b^{Y S T D}$ & $b$ & $b^{Y S T D}$ \\
\hline$X$ & 0.444 & 0.406 & 0.495 & 0.406 \\
\hline $\mathrm{Z}$ & & & 0.496 & 0.407 \\
\hline $\begin{array}{l}\text { Estimated variance of } \mathrm{Y}^{*} \\
\text { according to }(7 \mathrm{~b})\end{array}$ & \multicolumn{2}{|c|}{1.195} & \multicolumn{2}{|c|}{1.489} \\
\hline
\end{tabular}

